(C)2010 IEEE. Personal use of this material is permitted. However, permission to reprint/republish this material for advertising or promotional purposes or for creating new collective works for resale or redistribution to servers or lists, or to reuse any copyrighted component of this work in other works must be obtained from the IEEE. 


\title{
Challenges and methodologies in Passive Ocean Acoustic Tomography: An approach based on ICA
}

\author{
A. MANSOUR, IEEE Senior Member \\ ECE depart., Curtin University \\ Perth, WA 6152, Australia. \\ mansour@ieee.org, http://ali.mansour.free.fr
}

March 5, 2010

\begin{abstract}
The main idea of this manuscript consists on applying signal processing methods along with statistical approaches to achieve the characterization of a complex and hostile environment such as the oceanic underwater environment. In order to study, characterize (i.e. the measurement of the pressure, the salinity, the temperature, etc.) and extract the topographical features (depth, etc), Ocean acoustic tomography methods are widely used.

For many reasons, Passive ocean Acoustic Tomography (PAT) configuration becomes more attractive than the classic active one. However the new passive configuration is more challenging. In our project, some of the facing problems have been addressed. In this manuscript, major problems are described and a new separation scheme based on modified ICA algorithms is proposed. Finally, our experiment results corroborate the effectiveness of our proposed approach.
\end{abstract}

KEY WORD: Underwater acoustic applications, passive acoustic tomography, second order statistics in frequency domain, multipath channel, sparseness or non-stationary signals.

\section{Introduction}

It is well known that the oceans' ecosystem has a major impact on the global earth's ecosystem. In order to monitoring the marine ecosystem, many approaches have been previously proposed. The main common factor of these methods consists on the direct measurement of various environmental parameters (i.e. salinity, temperature, pressure, etc) using appropriate sensors. These approaches generally suffer from high cost and fail to provide adequate large scale monitoring. To solve these problems, researchers from all around the world are using underwater acoustic devices (as sonar) and technology along with powerful signal processing algorithms (classic and adaptive filtering, identification, clustering, etc.). Therefore, Acoustic Oceanic Tomography are widely used in many civil or military applications such as: Mapping underwater surfaces, oceanographical, meteorological applications (to measure the temperature, the salinity, the motion and the depth of the water), to improve sonar technology, so on. Many algorithms have been developed to deal with active acoustic tomography, $[17,16]$.

Active tomography strongly relies on the possibility to emit powerful acoustic signals in the ocean. Major problems can arise. Powerful emissions need heavy power supply which can drastically limit the efficiency of autonomous monitoring systems. It is well known that high power emission can cause drastic damages on marine mammals and disturb their behavior. Finally in a warfare context, some constraints about covertness may exit in the acoustic process. To overcome these problems, the concept of Passive Acoustic Tomography (PAT) has recently emerged in the community [21].

The main drawbacks of PAT are the lack of information about the number, the positions and the natures of the emitted signals. With more than two sources many actual tomography algorithms can't give satisfactory results [15].

This paper deals with the application of ICA algorithms in PAT in order to improve and simplified the PAT algorithms as well as the processing of the received signals. 


\section{Assumptions and Back- ground}

\subsection{Underwater Acoustic Channel}

According to [1], the sound speed $C(\mathrm{~m} / \mathrm{s})$ in the ocean is an increasing function of temperature $T$ $\left({ }^{\circ} \mathrm{c}\right)$, salinity $S$ (in Parts Per Thousand, ppt), and pressure, the latter being a function of depth $D$ (in meters) :

$$
\begin{aligned}
C= & 1449+4.6 T-0.05 T^{2}+2.3 \times 10^{-4} T^{3} \\
& +1.34(S-35)+0.02 D+1.6 \times 10^{-} 7 D^{2} \\
& -0.01 T(S-35)-7 \times 10^{-13} T D^{3}
\end{aligned}
$$

The above equation is empirical relationship which is satisfied when $0 \leq T \leq 30,30 \leq S \leq 40$, and $D \leq 8000$. Since most of these later parameters depend on time as well as geographic positions and hydrographic properties of the sea, we consider a simplified model where the sound propagation speed is assumed to be quite constant. This assumption can be satisfied in some underwater channels [5] (where the depth is about few hundred meters 200 to 300 , the emitters and the receivers are not so close to the water surface nor to the bottom, and the distances among emitters and receiver are less than 2 to $3 \mathrm{Km})$.

The reflected acoustic waves on the bottom of the propagation channel depend on many parameters such as the constitution and the geometrical properties of the bottom [4]. In our model a standard sand bottom has been considered and random coefficients have been added to characterize the other unknown parameters. The reflected acoustic waves on the top of the propagation channel, i.e. the water surface, depend also on many parameters such as the wind, the wave frequency as well as the swell properties [4]. For this reason, the water surface can't be considered as a flat surface. Therefore the direction of the reflected acoustic wave is dispersed in the space. However in average term, the reflected acoustic wave can be considered as obtained by a flat surface with some absorption coefficients [5]. In our model a flat surface has been considered and random coefficients have been added to characterize other unknown parameters.

Finally, an acoustic propagation model proposed by Schulkin [20] is considered. According to that model, the received signal should be multiplied by a corrective coefficient $p$ :

$$
p=\frac{1}{r} \exp \left(-\frac{\alpha r}{20}\right)
$$

here $r$ is the propagation distance and $\alpha$ stands for the Rayleigh absorption coefficient which it can be approximated by the following equation, [20]:

$$
\alpha=\left(1-6.54 * 10^{-4} * P_{w}\right)\left(\frac{S A f_{T} f^{2}}{f^{2}+f_{T}^{2}}+\frac{B f^{2}}{f_{T}}\right)
$$

where $f_{T}=21.9 * 10^{\left(6-\frac{1520}{T+273}\right)}$ (in $\left.\mathrm{kHz}\right), T$ is the water temperature $\left({ }^{\circ} \mathrm{C}\right), S=3.5 \%$ is the water salinity (in the ocean $S \approx 35 \mathrm{~g} / \mathrm{l}$ ), $P_{w}$ is the water pressure (in $\mathrm{kg} / \mathrm{m}^{2}$ ), $A=2.34 * 10^{-6}$ and $B=3.38 * 10^{-6}$.

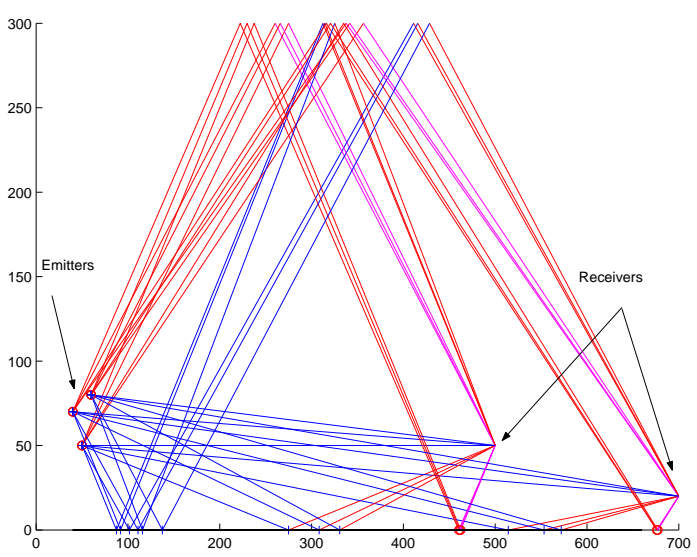

Figure 1: The obtained simulated acoustic channel parameters depend mainly on the channel depth, number of surface and bottom reflexions, emitters and receivers number, distances among receivers and emitters, sampling frequency, etc.

We should mention that the considered channel model is far from being a general and real one. Even though, the obtained channels can be characterized by a long (i.e. filter orders are up to 2500) and sparse (most of filter coefficients vanish) impulse response. The modified and simplified channels are still very challenging. In our future work, more realistic model will be taken into consideration.

\subsection{Basic features of Acoustic Source Signals}

In a passive acoustic tomography scenario, the source signals are signals of opportunities, i.e.:

- Natural signals: wave noise, earthquake, etc.

- Animals' signals: Motion noise (the prawns or shoal of fish motion noise) activity noise (Alpheus armatus, Red Snapping Shrimp, can produce can produce a strong snapping sound as a self-defence or to catch their prey, etc.), animals' sound (as the dauphines' and whales' sound, etc.)

- Artificial signals due to human activities (sonar, submarine or ship noise, etc.) 
In [2], an extensive experimental study has been conducted in order to classify and characterize many recorded anthropogenic signals (made by human activities) and natural signals (mainly animals sounds or natural noises). According to that study, one should take into consideration the following features $^{1}$ :

- Recorded signals are affected by a backgroun ocean noise which can be considered as an Ac ditive White Gaussian Noise (AWGN).

- Many signals are Gaussian or they have a ver weak kurtosis [10].

- Almost all of the signals are non-stationary si nals with more or less cyclic behavior as boc noises.

- Natural signals are very sparse ones and artif cial ones are very noisy.

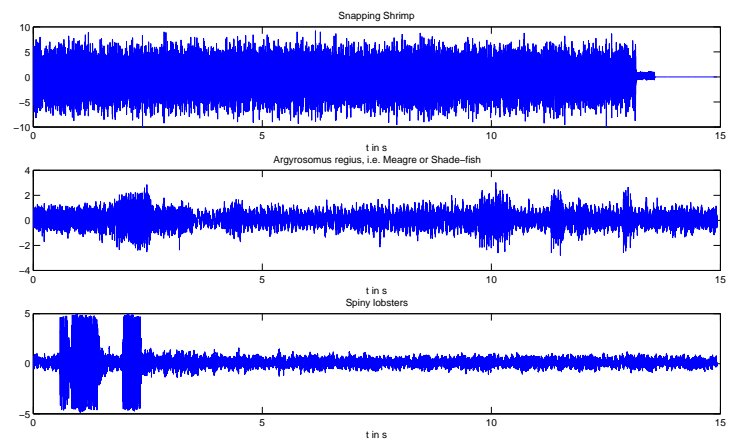

Figure 2: Natural acoustic signals

In order to characterize the typical and the most common signals, we conducted another study based on statistical criteria and using various ICA algorithm $[9,6]$. According to the latest study, The signals have various properties such as spatial diversity, different probability density functions (pdf), different temporal or spectral structures, different time-frequency signatures, etc :

- Most of signals are transitory signals with some distribution close to Gaussian ones.

- The sources have very inhomogeneous power (the power ratio can be up to a dozen of $\mathrm{dB}$ ).

- Signal to Noise Ratio (SNR) can be very limited depending on the operational situation.

- Even though ICA algorithms can handle convolutive mixtures. However, in our applications, the channel filter orders can be up to

\footnotetext{
${ }^{1}$ Only, the main relevant features to our application are mentioned.
}

few thousand. At the same time, such filter is very sparseness one. In fact, just few filter parameters do not vanish.

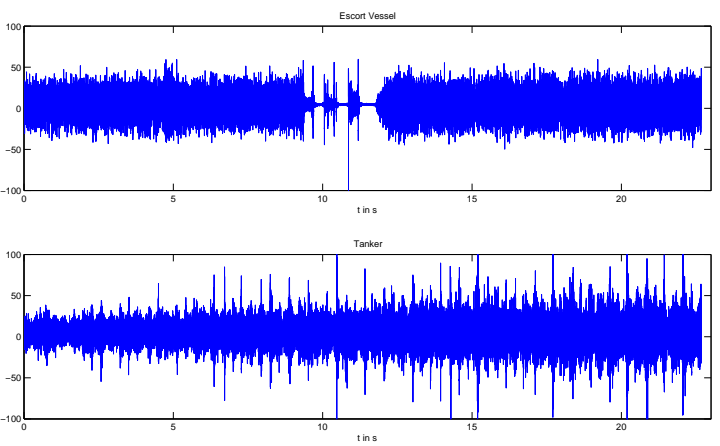

Figure 3: Artificial acoustic signals

In PAT applications, simple and cheap systems are often used which means that linear multi-sensor antenna are not recommended. Mainly, for this reason, ICA algorithms [?] can be used as a preprocessing stage to separate the received mixed signals into a set of independent and simple signals. This stage becomes an essential one, when a real life PAT scenario is considered. This pre-processing stage can simplify the task of the required acoustic tomography algorithms. In fact ICA algorithms can successfully handle Multi-Input Multi-Output (MIMO) channel. However, a real scenario of warfare applications makes the use of any ICA algorithm becomes very challenging. In fact, our experimental results show that many ICA algorithms couldn't give satisfactory results. The above mentioned properties have been considered to select and modified appropriate ICA algorithms [8].

\subsection{High order statistic estimator}

Many ICA algorithms or performance indexes, this point will be addressed afterward, are based on high order statistics. As we previously mentioned, the acoustic source signals are strongly non-stationary signals. In this case, the estimation of high order statistics becomes a highly challenging problem. For this reason, we previously proposed new high order statistic estimators adapted to our acoustic signals, further details can be found in $[13,14]$. 


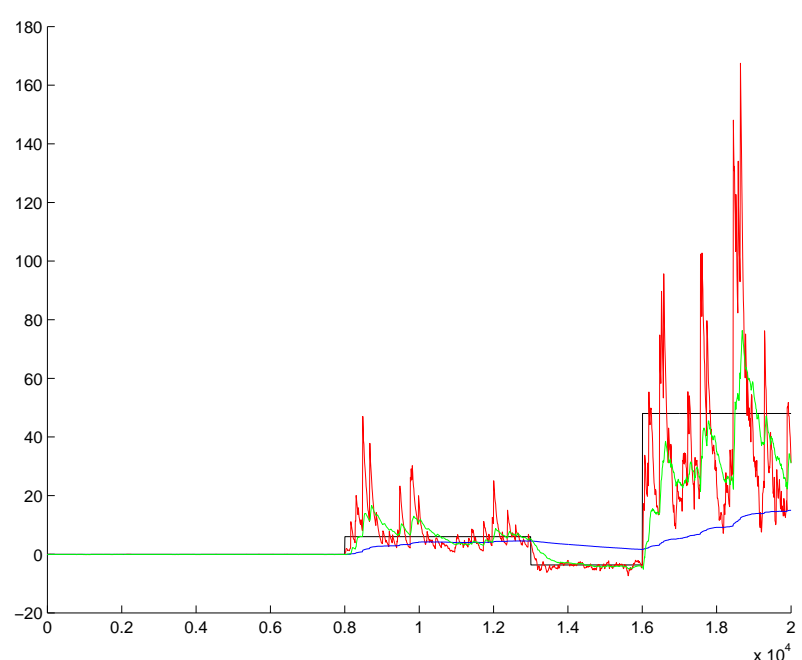

Figure 4: Estimation of cumulant3x1: The theoretical value is given in black, the classical estimator given in red, the new proposed estimators are in blue and in green.

\subsection{Source Number}

It has been already mentioned that the number, the positions and the features of the source signals are unknown. The channel parameters are strongly affected by the positions of the sources. This point has been discussed in section 2.1. The main features of the signals are also been discussed in the previous two subsections. It is well known that the number of sources is an important parameters for many ICA algorithms. We should mention that some ICA algorithms can roughly estimate that number. However the estimation results are satisfactory in our application.

Using the singular value decomposition of specific matrix along with some filter estimation algorithms, we proposed estimators of the source number [6]. This study is beyond the scope of the manuscript and it will be addressed in a future publication.

\subsection{Performance indexes}

Nowadays, a big number of ICA algorithms can be found in the literature [11]. Most of these algorithms have been proposed to deal with specific signals or channels in various applications [12]. In the case of similar applications, the choice of an appropriate algorithm can be easily done. Obviously, an appropriate selected algorithm should give the best experimental results. We should mention here that the acoustic signals aren't intelligible signals and they are strongly non-stationary signals. In addition, the underwater acoustic channel can be modeled by high order sparse filters. These prop-

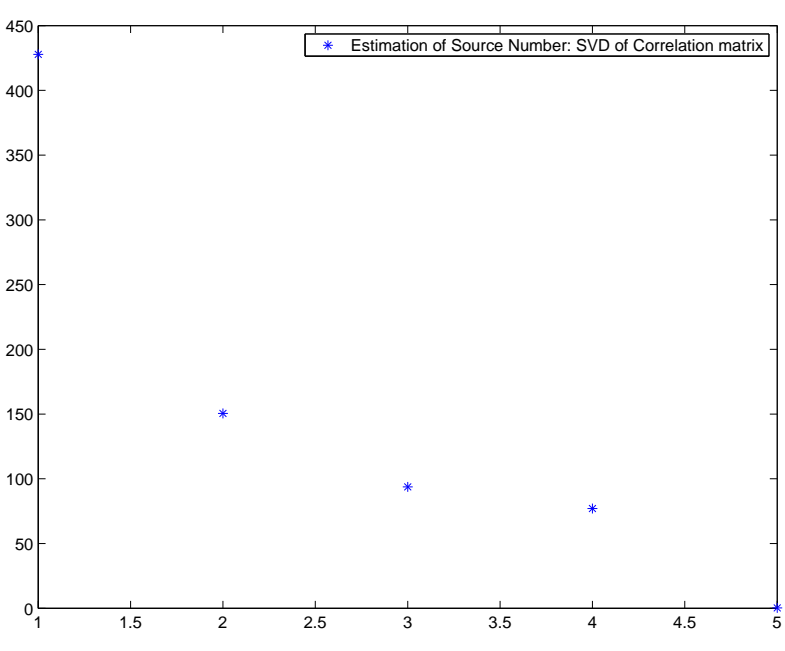

Figure 5: Estimation of source number using 5 observed signals and 4 acoustic sources.

erties means that an objective comparison among the algorithms becomes very hard. For this reason, we conducted a study on the available performance indexes. In that study, a set of modified or new performance indexes is proposed, further details can be obtained in [7].

\section{Mathematical Model}

Under some mild assumptions (i.e. Multiple Input Multiple Output configuration and ray propagation model) [3], acoustic underwater channel can be considered as multiple paths which, in frequency domain, each of them can be defined by a complex constant gain. Let $S(n)$ denotes a vector of $p$ unknown sources which are statistically independent from each other. $X(n)$ is a $q \times 1$ observed vector. The relationship between $S(n)$ and $X(n)$ is given by:

$$
X(n)=[\mathcal{H}(z)] S(n)+N(n)
$$

where $\mathcal{H}(z)$ stands for the channel effect.

\section{ICA Algorithms Applied to Acoustic Signals}

In our study, many ICA algorithms have been implemented and tested. Almost all of the tested algorithms can't perform well. For this reason, a complete separation structure has been implemented using pre- and post-processing modules of the signals, see fig. 6 or [8] for further details.

The two selected algorithms are: 


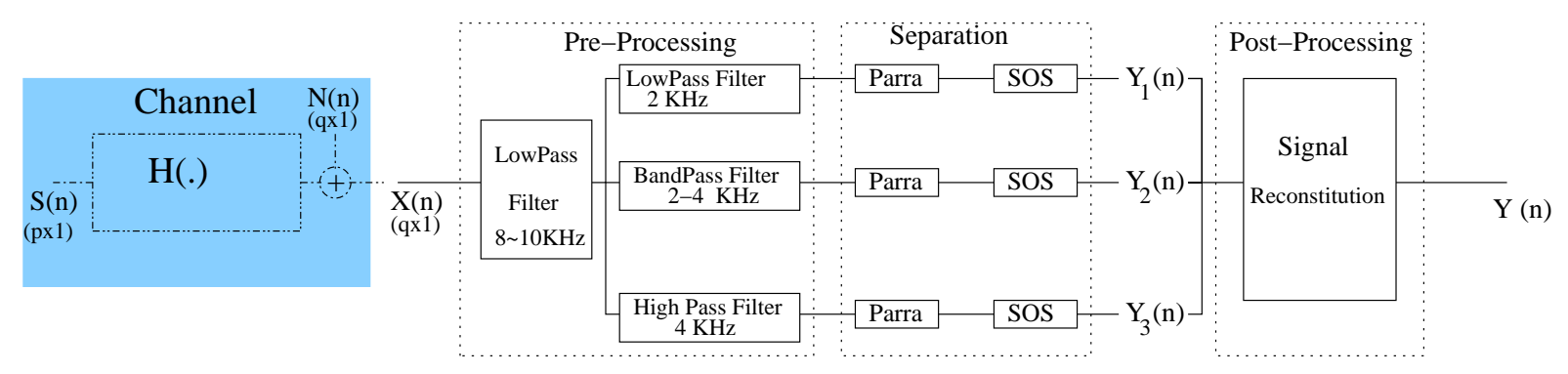

Figure 6: General Structure.

- A Frequency domain method for BSS of convolutive audio mixture (SOS) proposed by K. Rahbar et al. in [19].

- Convolutive Blind Separation of NonStationary Sources proposed by Parra et al. in [18].

\section{$5 \quad$ Experimental results}

Using the structure proposed in Fig. 6, many simulations have been conducted. Generally, over 500000-1000000 samples are needs to achieve the separation. The original sources are sampled at $44 \mathrm{KHz}$. In almost all the simulations, The separation of artificial or natural signals have been successfully achieved. Fig. 7 shows us different experimental results obtained by the different algorithms (Parra, SOS or Parra + SOS), each point corresponds to results of random simulations using Parra, SOS or Parra \& SOS algorithms. In this figure, a normalized positive performance index based on a nonlinear decorrelation is used [7]. The normalized performance index is forced to be zero for the mixture values and 1 for the sources.

\section{Conclusion}

In this manuscript, major problems facing the application of ICA algorithms in the Passive Acoustic Tomography (PAT) has been presented. Many simulations have been conducted and experimental studies show the necessity of considering preprocessing and post processing of the observed signals in order to achieve properly the separation of the sources. Many raised points have been solved. However, the obtained results can still be improved using more appropriate models which require more resources and computational efforts. These points will be the goal of our future studies.

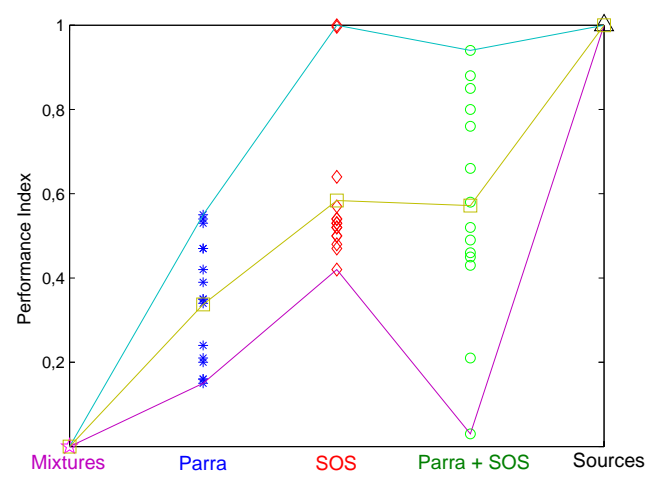

Figure 7: Experimental results obtained by the different algorithms (Parra, SOS or SOS + Parra) on divers configurations and using a normalized performance index (here the three curves represent maximum, minimum and average performance levels)

\section{References}

[1] P. Etter, Underwater acoustic modeling principles, techniques and applications. New York: Elsevier, 1991.

[2] D. Gaucher, C. Gervaise, and H. LE Flock, "Contributions to passive acoustic oceanic tomography," in 7ème Journées d'Acoustique Sous-Marine, Brest, France, 19-20 October 2004.

[3] C. Gervaise, A. Quinquis, and N. Martins, "Time frequency approach of blind study of acoustic submarine channel and source recognition," in Physics in Signal and Imane Processing, PSIP 2001, Marseille, France, January 2001.

[4] B. L.M. and L. Y.P., Fundamentals of ocean acoustics. New York: Springer Verlag, 2003.

[5] X. Lurton, Introduction to underwater acoustics principles and applications. London: Springer, 2002.

[6] A. Mansour, "Rapport final de l'étude 6.1 : séparation de sources du poste 6 : fusion pour la diversité d'information et l'assimilation de données," ENSIETA, Tech. Rep., January 2005, projet Méthodes d'Observations Discrètes de l'Environnement (MODE). 
[7] A. Mansour and A. Al falou, "Performance indexes of BSS for real world applications," in XIVth European Signal Proc. Conf EUSIPCO-06, Florence, Italy, Septembre 2006.

[8] A. Mansour, N. Benchekroun, and C. Gervaise, "Blind separation of underwater acoustic signals," in 6th International Workshop on Independent Component Analysis and blind Signal Separation, ICA2006, Charleston, SC, USA, March 5-8 2006.

[9] A. Mansour and C. Gervaise, "Ica applied to passive ocean acoustic tomography," WSEAS Trans. on Acoustics and Music, vol. 1, no. 2, pp. 83-89, April 2004.

[10] A. Mansour and C. Jutten, "What should we say about the kurtosis?" IEEE Signal Processing Letters, vol. 6, no. 12, pp. 321-322, December 1999.

[11] A. Mansour, A. Kardec Barros, and N. Ohnishi, "Blind separation of sources: Methods, assumptions and applications." IEICE Transactions on Fundamentals of Electronics, Communications and Computer Sciences, vol. E83-A, no. 8, pp. 14981512, August 2000.

[12] A. Mansour and M. Kawamoto, "Ica papers classified according to their applications \& performances." IEICE Transactions on Fundamentals of Electronics, Communications and Computer Sciences, vol. E86-A, no. 3, pp. 620-633, March 2003.

[13] A. Martin and A. Mansour, "Comparative study of high order statistics estimators," in 2004 International Conference on Software, Telecommunications and Computer Networks, Split (Croatia), Dubrovnik (Croatia), Venice (Italy), October 1013 2004, pp. 511-515.

[14] — , "High order statistic estimators for speech processing," in ITST 2005 - 5th International Conference on ITS Telecommunications, Brest, France, Juin 27-29 2005.

[15] N. Martins, S. Jesus, C. Gervaise, and A. Quinquis, "A time-frequency approach to blind deconvolution in multipath underwater channels," in Proceedings of International Conference on Acoustics Speech and Signal Processing 2002, ICASSP 2002, Orlando, Florida, U.S.A, 13-17 May 2002.

[16] W. Munk, P. Worcester, and C. Wunsch, Ocean Acoustic Tomography. Cambridge: Cambridge University Press, 1995.

[17] W. Munk and C. Wunsch, "Ocean acoustic tomography: A scheme for large scale monitoring," Deep Sea Research, vol. 26, pp. 123-161, 1979.

[18] L. Parra and C. V. Alvino, "Convolutive blind separation of non-stationnary sources," IEEE Trans. on Speech and Audio Processing, vol. 8, no. 3, pp. 320-327, May 2000.

[19] K. Rahbar, J. Reilly, and J. H. Manton, "Blind identification of mimo fir systems driven by quasistationary sources using second order statistics: A frequency domain approach," IEEE Trans. on Signal Processing, vol. 52, no. 2, pp. 406-417, February 2004.
[20] M. Shulkin and H. W. Marsh, "Sound absorption in sea water," Journal of the Acoustical Society of America, vol. 134, pp. 864-865, 1962.

[21] S. Vallez, A. Martin, A. Mansour, C. Gervaise, and A. Khenchaf, "Contribution to passive acoustic ocean tomography- part IV: A data fusion strategy for blind source separation and classification," in IEEE Conference and Exhibition OCEANS'05 Europe, Brest, France, 2005. 